\title{
ApoG2 induces cell cycle arrest of nasopharyngeal carcinoma cells by suppressing the c-Myc signaling pathway Zhe-Yu Hu${ }^{1}$, Jian Sun ${ }^{1}$, Xiao-Feng Zhu ${ }^{1}$, Dajun Yang ${ }^{2}$ and Yi-Xin Zeng*1
}


Guangzhou, PR China and ${ }^{2}$ Ascenta Therapeutics Incorporation, Malvern, Pennsylvania, USA

Email: Zhe-Yu Hu - huzheyu24@gmail.com; Jian Sun - denzel@21cn.com; Xiao-Feng Zhu - zhuxfeng@mail.sysu.edu.cn; Dajun Yang - dyang@Ascenta.com; Yi-Xin Zeng* - zengyix@mail.sysu.edu.cn

* Corresponding author

Published: 23 August 2009

Journal of Translational Medicine 2009, 7:74 doi:10.1 I86/1479-5876-7-74
Received: I June 2009

Accepted: 23 August 2009

This article is available from: http://www.translational-medicine.com/content/7/I/74

(C) 2009 Hu et al; licensee BioMed Central Ltd.

This is an Open Access article distributed under the terms of the Creative Commons Attribution License (http://creativecommons.org/licenses/by/2.0), which permits unrestricted use, distribution, and reproduction in any medium, provided the original work is properly cited.

\begin{abstract}
Background: apogossypolone (ApoG2) is a novel derivate of gossypol. We previously have reported that APOG2 is a promising compound that kills nasopharyngeal carcinoma (NPC) cells by inhibiting the antiapoptotic function of Bcl-2 proteins. However, some researchers demonstrate that the antiproliferative effect of gossypol on breast cancer cells is mediated by induction of cell cycle arrest. So this study was aimed to investigate the effect of ApoG2 on cell cycle proliferation in NPC cells.

Results: We found that ApoG2 significantly suppressed the expression of c-Myc in NPC cells and induced arrest at the DNA synthesis (S) phase in a large percentage of NPC cells. Immunoblot analysis showed that expression of c-Myc protein was significantly downregulated by ApoG2 and that the expression of c-Myc's downstream molecules cyclin DI and cyclin E were inhibited whereas $\mathrm{p} 2 \mathrm{I}$ was induced. To further identify the cause-effect relationship between the suppression of c-Myc signaling pathway and induction of cell cycle arrest, the expression of c-Myc was interfered by siRNA. The results of cell cycle analysis showed that the downregulation of cMyc signaling pathway by siRNA interference could cause a significant arrest of NPC cell at $S$ phase of the cell cycle. In CNE-2 xenografts, ApoG2 significantly downregulated the expression of c-Myc and suppressed tumor growth in vivo.

Conclusion: Our findings indicated that ApoG2 could potently disturb the proliferation of NPC cells by suppressing c-Myc signaling pathway. This data suggested that the inhibitory effect of ApoG2 on NPC cell cycle proliferation might contribute to its use in anticancer therapy.
\end{abstract}

\section{Background}

Nasopharyngeal carcinoma (NPC) is an epithelial squamous cell carcinoma endemic in Southeast Asia and parts of Mediterranean and northern Africa [1]. Radiotherapy alone cures more than $90 \%$ of cases of stage I NPC; however, patients with advanced disease tend to experience therapy failure. Several groups have shown that the 5-year survival rate for concurrent chemotherapy and radiotherapy is higher than that for radiotherapy alone in patients with advanced disease [2,3]. Currently, cisplatin combined with 5-fluorouracil is the first-line chemotherapeutic regimen for NPC. Although this regimen has manageable toxic effects and has yielded response rates ranging from $65 \%$ to $75 \%[4]$, an urgent need for inpa- 
tient administration of chemotherapy has accelerated the development of newer, more tolerable and potent platinum-based regimens. We previously showed that ApoG2 in particular could potently kill NPC cells and had a synergic effect with cisplatin to induce cell death [5]. In this study, we further investigated the effect of ApoG2 on cell cycle regulator proteins and cell cycle progression.

Gossypol and its derivates reportedly induce apoptosis by inhibiting the antiapoptotic function of the Bcl-2 family of proteins $[5,6]$. Also, authors have found cell cycle arrest in gossypol-treated cells. Several cell cycle-related molecules are involved in gossypol-induced cell cycle arrest. For example, researchers have reported that gossypolinduced cell death was coupled with upregulation of c-Fos expression and biphasic c-Myc expression in rat spermatocytes [7]. Furthermore, transforming growth factor- $\beta$ is activated by gossypol in prostate cancer cells, and gossypol upregulates p21 expression and downregulates cyclin $\mathrm{D} 1$ and $\mathrm{Rb}$ expression in colon cancer cells $[8,9]$. Modifications of these cell cycle-related molecules result in cancer cell arrest at G0/G1 phase of the cell cycle. However, Chang et al. found that gossypol did not affect cell cycle progression or the $\mathrm{p} 53$ or $\mathrm{p} 21$ /WAF signaling pathway in A549 human alveolar lung cancer cells [10]. Different oncogenic pathways are activated in different types of cancer, and treatment with gossypol may have various biochemical and molecular impacts on different cancers with specific biological behaviors.

NPC is associated with Epstein-Barr virus (EBV) infection and genetic susceptibility. EBV-encoded latent membrane protein 1 (LMP1) is a principal oncogene in cases of NPC; it can activate a number of signaling pathways, including nuclear factor- $\mathrm{kb}$, mitogen-activated protein kinase, and phosphoinositide 3-kinase [11]. Besides the LMP1induced oncogenic pathways, dysregulation of factors such as p16, cyclin D1, and cyclin E leads to aberrations in the cell cycle in NPC cells. Therefore, NPC has multiple unique abnormalities that are potential targets for novel treatments. In this study, we examined the effect of ApoG2 on cell cycle distribution and the involved signal pathways in NPC cells. The results demonstrated that ApoG2 potently arrested cells at $S$ phase of the cell cycle. We also observed that suppression of the c-Myc signaling pathway was responsible for the ApoG2-induced cell cycle arrest.

\section{Materials and methods Cells, Drugs, and Reagents}

Poorly differentiated human NPC cell lines CNE-2 and HONE-1 were originally obtained from NPC patients and maintained in our laboratory in RPMI-1640 (Gibco/BRL, Gaithersburg, MD) supplemented with $10 \%$ heat-inactivated fetal bovine serum (Thermo Scientific HyClone,
Logan, UT). Cells were incubated in a humidified $5 \% \mathrm{CO}_{2}$ atmosphere at $37^{\circ} \mathrm{C}$. ApoG2, which was supplied by Dajun Yang (Ascenta Therapeutics Incorporation, Malvern, Pennsylvania), was dissolved in pure dimethyl sulfoxide (DMSO) at the stock concentration of $20 \mathrm{mmol} / \mathrm{l}$ and stored at $-20^{\circ} \mathrm{C}$. 3-(4,5 dimethylthiazol-2-yl)-2, 5-diphenyltetrazolium (MTT) were purchased from SigmaAldrich (St. Louis, MO). In in vivo experiments, for intraperitoneal (i.p.) injection, ApoG2 was suspended in 0.5\% sodium carboxymethylcellulose and prepared on the day of use.

\section{MTT Assay}

NPC cell viability was assessed using an MTT assay based on mitochondrial conversion of MTT from soluble tetrazolium salt to an insoluble colored formazan precipitate, which was dissolved in DMSO and quantitated using a spectrophotometer (Thermo Multiskan MK3; Thermo Fisher Scientific, Waltham. MA) with optical density (OD) values [12]. NPC cells were plated in 96-well culture clusters (Costar, Cambridge, MA) at a density of 15,000 to 25,000 cells $/ \mathrm{ml}$. Serial dilutions of ApoG2 were prepared from a stock solution to the desired concentrations. The final DMSO concentration was less than $0.1 \%(\mathrm{v} / \mathrm{v})$. All experimental concentrations of ApoG2 were prepared in triplicate. Cells were treated with ApoG2 for 24, 48 and 72 $\mathrm{h}$. Before termination of treatment, cells were incubated with $10 \mu \mathrm{l}$ of $10 \mathrm{mg} / \mathrm{ml}$ MTT for $4 \mathrm{~h}$. Then MTT and medium were depleted, and $100 \mu \mathrm{l}$ of DMSO was added to the plates. The percent absorbance of Apog2-treated cells relative to the control (DMSO treated cells, DMSO concentration was less than $0.1 \%$ ) was plotted as a linear function of the drug concentration. The antiproliferative effect of ApoG2 on NPC cells was measured as the percent of viable cells relative to the control using the equation $100 \% \times \mathrm{OD}_{\mathrm{T}} / \mathrm{OD}_{\mathrm{C}}$, in which $\mathrm{OD}_{\mathrm{T}}$ is the mean $\mathrm{OD}$ value of the ApoG2-treated treated samples and $\mathrm{OD}_{\mathrm{C}}$ is the mean OD value of the control samples. The $50 \%$ inhibitory concentration of ApoG2 was defined as the concentration of the drug required to achieve $50 \%$ growth inhibition relative to control populations.

\section{Cell Cycle Analysis}

Untreated control and ApoG2-treated CNE-2 cells were harvested, washed twice with phosphate-buffered saline (PBS), and fixed dropwise with $2 \mathrm{ml}$ of $70 \%$ ice-cold ethanol. After cells fixed overnight at $4{ }^{\circ} \mathrm{C}$, cells were then washed twice with PBS; cells were then incubated in RNase $(20 \mu \mathrm{g} / \mathrm{ml})$ at $37^{\circ} \mathrm{C}$ for $30 \mathrm{~min}$ to avoid staining the RNA. Next, the cells were washed once with PBS; PI was added to samples at a final concentration of $15 \mu \mathrm{mol} /$ 1 , and after 5 min of incubation, the cells were analyzed using flow cytometry (Beckman Coulter, Fullerton, CA). The percentages of the nuclei in CNE-2 cells at each phase of the cell cycle (G1, S, G2/M) were calculated using the 
MultiCycle software program (Phoenix Flow Systems, San Diego, CA).

\section{Immunoblot Analysis}

Protein analysis using immunoblotting and immunoprecipitation was performed with primary antibodies against p53 (sc-126; Santa Cruz Biotechnology, Santa Cruz, CA), p21 (sc-6246; Santa Cruz Biotechnology), c-Myc (sc-42; Santa Cruz Biotechnology), cyclin E (sc-481; Santa Cruz Biotechnology), cyclin D1 (sc-8396; Santa Cruz Biotechnology), and actin (clone AC-15; Sigma-Aldrich) as described previously [13]. Total cell lysates were harvested, electrophoresed using $12 \%$ sodium dodecyl sulfate-polyacrylamide gel electrophoresis, and transferred to polyvinylidene difluoride membranes (Roche, Grenzacherstrasse, Basel, Switzerland). Immunoblotting was performed using the primary antibodies described above followed by detection of protein expression using secondary antibodies conjugated with horseradish peroxidase (Cell Signaling Technology, Danvers, MA), and blots were developed using ECL chemiluminescent reagent (Cell Signaling Technology).

\section{RNA Interference}

Transient small interfering RNA (siRNA) transfection was performed using Lipofectamine 2000 (Invitrogen, San Diego, CA) and $50 \mathrm{nM}$ siRNA oligonucleotides. Commercially purchased siRNAs (Ribobio, Guangzhou, People's Republic of China) were scrambled (nontargeting), glyceraldehyde-3-phosphate dehydrogenase siRNA, and cMyc siRNA. The three independent oligonucleotides designed for the c-Myc siRNA sequences were 5'CAGAAATGTCCTGAGCAAT-3', 5'-AAGGTCAGAGTCTGGATCACC-3', and 5'-AAGGACTATCCTGCTGCCAAG$3 '$. The siRNA duplexes were introduced into CNE-2 cells according to the siRNA manufacturer's protocol. After transfection with siRNA for $48 \mathrm{~h}$, cells were harvested for immunoblots and cell cycle analysis. The scrambled siRNA construct was used as a negative control.

\section{In vivo treatment and immunohistochemistry assay}

Four-week-old athymic nude (nu/nu) mice obtained from the Animal Center of Southern Medical University (Guangdong, China) received subcutaneous injection of 1 $\times 10^{7} \mathrm{CNE}-2$ cells in each axillary area. When subcutaneous tumors developed to more than $1,500 \mathrm{mg}$, mice were euthanized and tumors were dissected and mechanically dissociated into equal pieces to be transplanted into the flank areas of a new group of mice. When xenograft tumors became palpable (about $0.1 \mathrm{~mm}^{3}$ ), mice were randomly divided into control $(0.5 \%$ sodium carboxymethylcellulose solution) and ApoG2 $(120 \mathrm{mg} / \mathrm{kg}$ of body weight given by intraperitoneal injection daily) groups. Each group contained 8 mice, and there was no difference in tumor size between groups. Based on our lab's policy, when xenograft tumors developed to more than 1,000 $\mathrm{mg}$, mice were euthanized and tumors were dissected and weighed. Immunohistochemical analysis was performed on tissue-sample sections of CNE-2 xenografts obtained from control and ApoG2. All samples were stained with hematoxylin and eosin and microscopically examined to confirm the CNE-2 cell origin. Sections were then stained with c-Myc (\#; Santa Cruz) at $4{ }^{\circ} \mathrm{C}$ overnight and then visualized using diaminobenzidine (DAB) (DAKO Liquid $\mathrm{DAB}$, Dako, Carpinteria, CA) as peroxidase substrates.

\section{Statistical analysis}

All analyses to compare the significance of measured levels were completed using the unpaired $t$-test by SPSS 16.0 software.

\section{Results \\ ApoG2 Inhibits Cell Proliferation of NPC cells}

Our previous work demonstrated that ApoG2 (Fig. 1A, the chemical structure of ApoG2) could significantly kill NPC cells and suppress the growth of NPC xenografts in nude mice. In this study, we reevaluated the antiproliferative effect of ApoG2 on CNE-2 cells using an MTT assay. We treated CNE-2 cells with 5, 10 and $20 \mu \mathrm{M}$ ApoG2 for 24, 48 and $72 \mathrm{~h}$. This treatment resulted in dose- and timedependent inhibition of cell proliferation (Fig. 1B). At 10 and $20 \mu \mathrm{M}$, ApoG2 inhibited about $60 \%$ and $90 \%$ of the cell growth, respectively, at $72 \mathrm{~h}$.

Moreover, among four NPC cell lines C666-1 (EBV infected), CNE-1 (highly differentiated), CNE-2 (poorly differentiated) and HONE-1 (poorly differentiated), ApoG2 treatment resulted in a tremendous inhibition of cell proliferation in C666-1, CNE-1 and CNE-2 NPC cell lines. At $10 \mu \mathrm{M}$, ApoG2 inhibited more than $60 \%$ of the cell growth of C666-1, CNE-1 and CNE-2 cells at $72 \mathrm{~h}$. In contrast, only about $30 \%$ of HONE- 1 cell proliferation was inhibited by $10 \mu \mathrm{M}$ ApoG 2 treatment for $72 \mathrm{~h}$.

\section{AapoG2 Treatment Induces NPC Cells Arresting in S Phase of Cell Cycle}

Gossypol reportedly induces cell cycle arrest in prostate cancer cells and colon cancer cells $[8,9]$. To determine whether ApoG2 could also induce cell cycle arrest in NPC cells, we performed a cell cycle analysis using flow cytometry. The results showed the same with our previous work [5] that, at $48 \mathrm{~h}$ after treatment, ApoG2 did not induce obvious cell apoptosis in NPC cells and little cells were accumulated in sub-G1 phase. Instead, ApoG2 induced cell cycle arrest at the DNA synthesis (S) phase in a large percentage of NPC cells at this time. More than $60 \%$ of C666-1, CNE-1 and CNE-2 cells were arrested at S phase at $48 \mathrm{~h}$ after exposure to 5 and $10 \mu \mathrm{M}$ ApoG2, whereas only 34\%, 39\% and 35\%, respectively, of untreated C666- 
A.

Apogossypolone (ApoG2)<smiles>CC1=C(C2=C(C)C(=O)c3c(cc(O)c(O)c3C(C)C)C2=O)C(=O)c2cc(O)c(O)c(C(C)C)c2C1=O</smiles>

B.

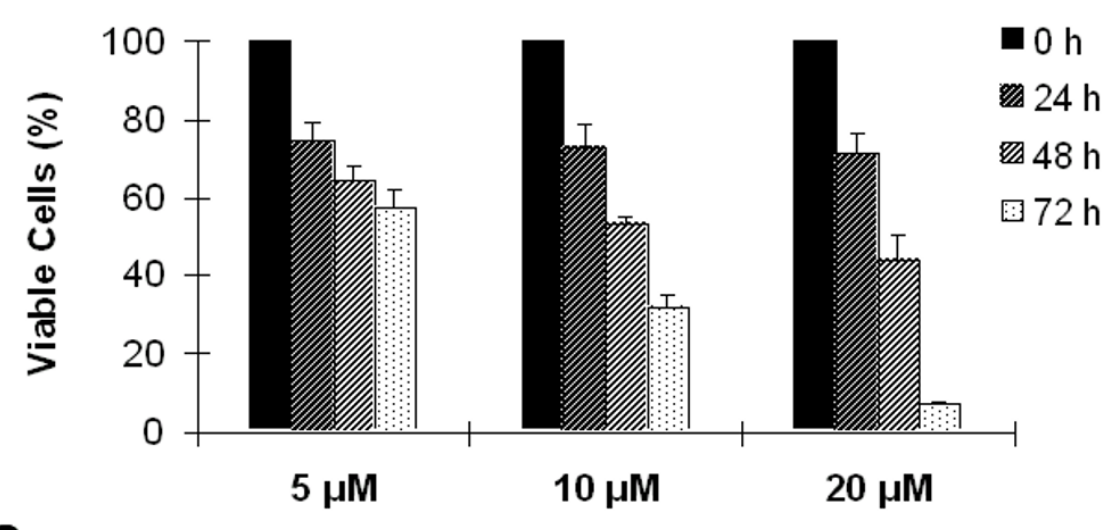

C.

Effect of ApoG2 on NPC cell lines



Figure I

ApoG2 and its inhibitory effect on CNE-2 cell proliferation. (A) The chemical structure of ApoG2. (B) Effect of ApoG2 on NPC cell survival. Cells were exposed to 5, 10, and $20 \mu \mathrm{M}$ ApoG2 for 24, 48, and $72 \mathrm{~h}$. Compared to control cells (treated with $0.1 \%$ DMSO), percentage of viable cells in treated samples was measured using an MTT assay (mean \pm standard deviation for three experiments). (C) The inhibitory effect of ApoG2 on four NPC cell lines (HONE-I, CNE-2, CNE-I and C666-I) was compared after 72-hr treatment. Points, average of three experiments; bars, SD. 
1, CNE-1 and CNE-2 cells were arrested at S phase (Fig. $2 \mathrm{~A}-\mathrm{C})$.

Because we observed that another NPC cell line, HONE-1, was much less sensitive to ApoG2 treatment and exhibited a much higher $50 \%$ inhibitory concentration value of ApoG2 (more than 10-fold) than C666-1, CNE-1 and CNE-2 cells (data not shown), we assessed the effect of ApoG2 on the cell cycle in this cell line. Treatment with 10 $\mu \mathrm{M}$ ApoG2 induced about $60 \%$ HONE-1 cells arresting at S phase (Fig. 2D); in comparison, only $34 \%$ of untreated HONE-1 cells were arrested at $S$ phase of the cell cycle. These data implied that ApoG2-induced cell cycle arrest is not correlated with the sensitivity of cells to ApoG2, because in both ApoG2-sensitive NPC cells and ApoG2insensitive HONE-1 cells, ApoG2 treatment could result in significant cell cycle arrest. These data also implied that ApoG2-induced cell cycle arrest was not caused the inhibition of Bcl-2 proteins and other molecular mechanisms might be involved in ApoG2-induced cell cycle arrest in NPC cells.

\section{Downregulation of c-Myc Expression Leads to Cell Cycle Arrest by ApoG2 in NPC cells}

Because researchers have reported that cell cycle-regulating molecules, such as p $21, \mathrm{p} 53$, and TGF- $\beta 1$, play roles in gossypol-induced cell cycle arrest $[9,14]$, we hypothesized that ApoG2 can also modify some cell cycle regulators, resulting in cell cycle arrest in NPC cells. Consistent with our hypothesis, treatment with $10 \mu \mathrm{M}$ ApoG2 significantly decreased the level of c-Myc protein expression at $24 \mathrm{~h}$ in CNE-2 cells (Fig. 3A). Moreover, expression of p21 protein was upregulated as early as $24 \mathrm{~h}$ and gradually returned to low level at $72 \mathrm{~h}$ since most of the CNE-2 cells were dead at this time (Fig. 3B); unlike p21, expression of both cyclin D1 and cyclin E were downregulated following the degradation of c-Myc. We observed no changes in p53 protein expression (Fig. 3B). Similar changes in the cMyc pathway were also detected in ApoG2-treated HONE1 cells (Fig. 3C), which was in agreement with the results of cell cycle analysis that ApoG2 induced cell cycle arrest in both sensitive CNE-2 cells and insensitive HONE-1 cells.

\section{Downregulation of c-Myc Expression by siRNA Leads to Cell Cycle Arrest at $S$ Phase in CNE-2 Cells}

Authors have reported that the oncoprotein c-Myc regulates the expression of p21 and cyclins, increases cyclin DCDK4 activity, and facilitates cell cycle progression [15]. Also, Fan et al. found that upregulated expression of c-Myc protein in NPC cells contributed to unrestricted cell proliferation, metastasis, and tumor progression [16]. In our study, the immunoblots data indicated that suppression of the c-Myc pathway might be responsible for ApoG2induced cell cycle arrest in NPC cells. To test this hypoth- esis, we used three siRNA oligonucleotides (Ribobio, Guangzhou, China) to knock down c-Myc protein in CNE-2 cells. As shown in fig. 4A, all these three oligonucleotides significantly suppressed the expression of c-Myc protein; the reduction in c-Myc expression led to upregulation of p21 expression and downregulation of cyclin D expression. Cell cycle analysis showed that incubation with scrambled siRNA resulted in a significantly lower CNE-2 cell population arrested at $S$ phase than did incubation with c-Myc siRNA (Fig. 4B and 4C). Compared to srambled siRNA, c-Myc siRNAs induced conspicuous increasing of cells in S phase in CNE-2 cells at $48 \mathrm{~h}$ (Fig. $4 \mathrm{D}$ ). Based on these results, we suggested that suppression of the c-Myc pathway by ApoG2 leads directly to cell cycle arrest in NPC cells.

ApoG2 inhibites c-Myc expression level in CNE-2 xenografts in nude miceTo assess the effect of ApoG2 on c-Myc expression in vivo, we used the CNE-2 xenografts nude mice model. When control xenografts developed to more than $1,000 \mathrm{mg}$, all mice were euthanized and tumors were dissected, weighed and fixed for immunochemistry assay. As shown in fig. $5 \mathrm{~A}$ and $5 \mathrm{~B}$, compared to NS (normal saline) treatment group, ApoG2 treatment provoked a significant reduction in c-Myc expression level in CNE-2 xenografts. Antitumor activities of ApoG2 (120 $\mathrm{mg} / \mathrm{kg}$ i.p. injection once every three days) against CNE-2bearing nude mice was measured by weighing the weight of CNE-2 xenografts (Fig. 5C). As shown in fig. 5D, compared to control treatment, ApoG2 could significantly inhibit tumor weight in CNE-2 xenografts $(\mathrm{p}<0.001)$.

\section{Discussion}

ApoG2 is the oxidation product of gossypol and has two aromatic hydrocarbon quinone groups. Authors have reported that aromatic hydrocarbon quinone stimulates ROS production in hepatic cells [17]. As we known, elevated ROS levels may damage cellular DNA, inducing generation of oxidized bases, DNA strand breaks, and stop of DNA replication, in ApoG2-treated CNE-2 cells. Recent studies provided evidence that multiple chemopreventive agents can cause generation of ROS to trigger signal transduction, culminating in cell cycle arrest and/or apoptosis $[18,19]$. However, Van Poznak et al. and Zhang et al. suggested that gossypol-induced cell cycle arrest is associated with alterations of p21, cyclin D1, and p53 and showed that $\mathrm{p} 21$ is the first target of gossypol to inhibit cell growth in vivo $[9,20]$. Our data indicated that ApoG2 induced massive cells arrest at $S$ phase of the cell cycle not only in ApoG2-sensitive NPC cells but also in ApoG2-insensitive HONE-1 cells (Fig. 3). Results of signaling pathway analysis showed that downregulation of c-Myc protein expression was the major upstream event in ApoG2-induced cell cycle arrest in NPC cells (Fig. 4). Basically, the effect of cMyc on cell cycle is to drive quiescent cells into the cell 
A.
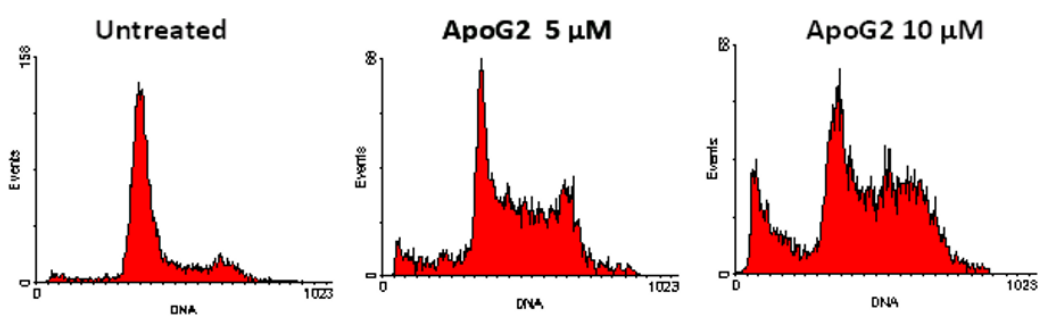

B.


C.
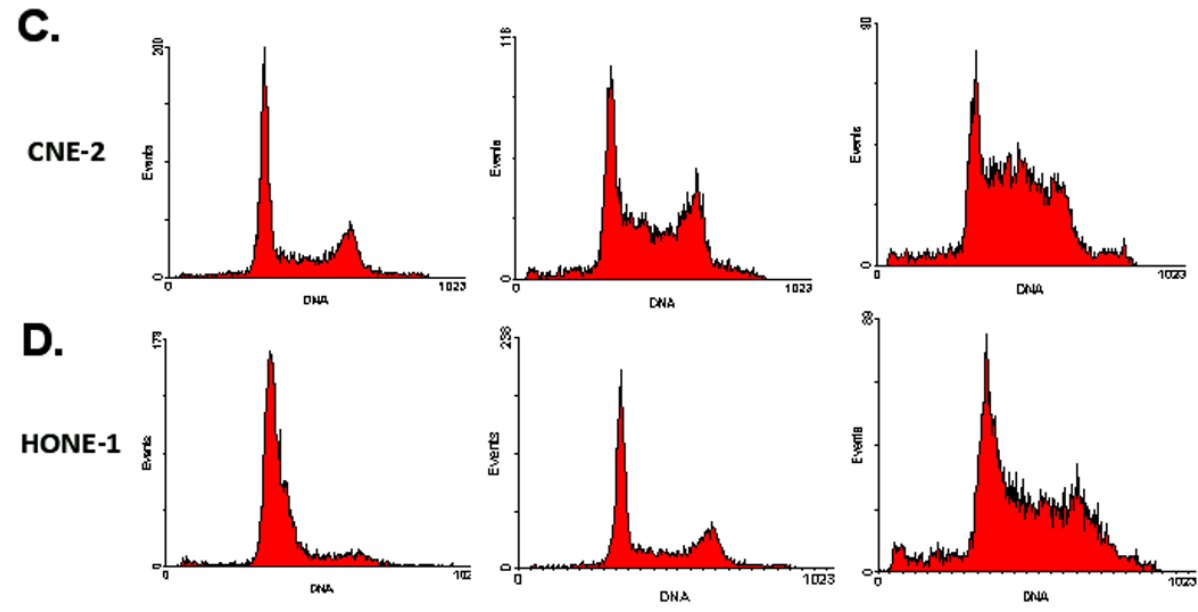

E.

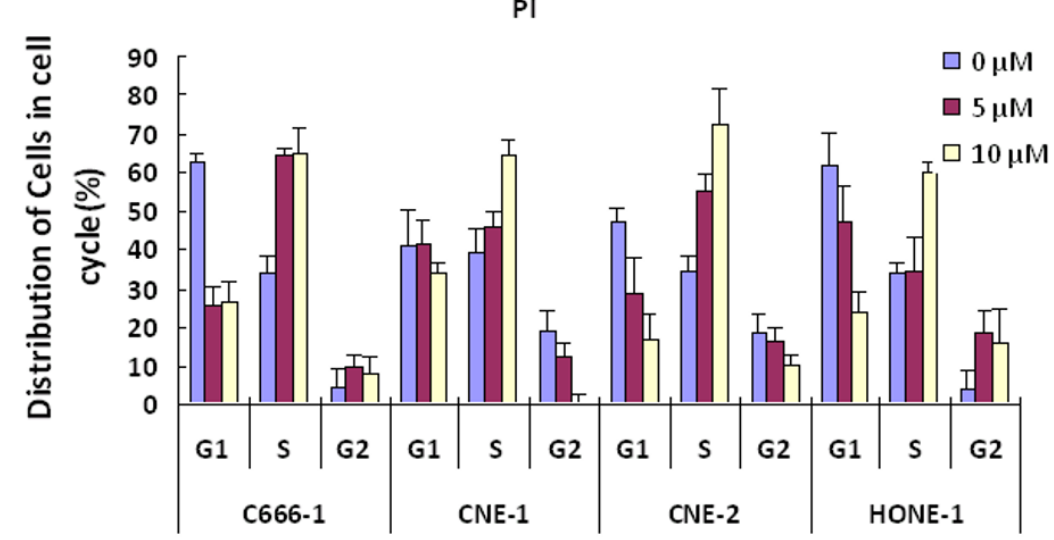

Figure 2

Arrest of NPC cells at S phase of the cell cycle by treatment with ApoG2. Arrest of NPC cells at S phase by ApoG2. C666-I (A), CNE-I (B), CNE-2 (C) and HONE-I (D) cells were treated with 5 and $10 \mu \mathrm{M}$ ApoG2 for 48 h. DNA cell cycle analysis was performed using PI staining and flow cytometry. Each histogram is representative of three experiments. (E) Cell cycle analysis showed that ApoG2 treatment induced a conspicuous increasing of cells in S phase in four NPC cell line at $48 \mathrm{~h}$. Bar heights, average of three independent experiments; bars, SD. 

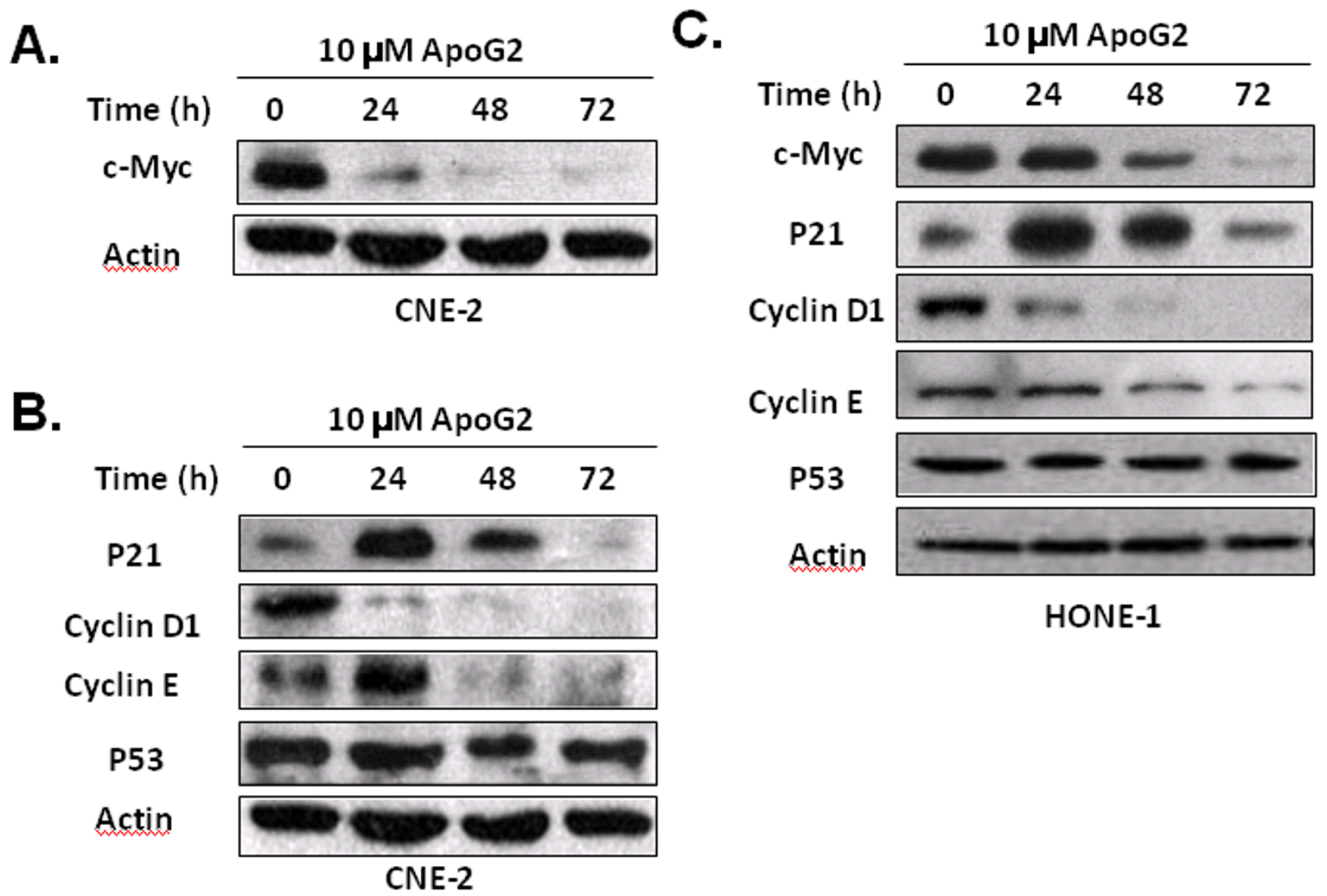

Figure 3

Treatment with ApoG2 induces alterations in the expression of c-Myc, p2 I, and cyclins. (A) The effect of ApoG2 on the expression of c-Myc. CNE-2 cells were incubated with $10 \mu \mathrm{M}$ ApoG2 for 24 to $72 \mathrm{~h}$, and cell lysates were analyzed using immunoblotting. (B) The effect of ApoG2 on the expression of molecules downstream from c-Myc. After treatment with ApoG2, CNE-2 cell lysates were analyzed using immunoblotting with anti-p2I, -cyclin DI, -cyclin E, and -p53 antibodies. (C) The effect of ApoG2 on cell cycle-regulatory molecules in HONE-I cells. Cells were treated with $10 \mu$ M ApoG2 for 24 to 72 $\mathrm{h}$, and cell lysates were analyzed using immunoblotting.

cycle, and shortening G1 and promoting S phase entry thereby. The down-regulation of c-Myc should cause a preferential G1/S arrest rather than $S$ arrest. However, in NPC cells, although p53 was highly expressed and its expression was never downregulated by ApoG2 in this study, p53 was mutated and functionally impaired by Epstein-Barr virus nuclear antigen 5 and deltaN-p63 in NPC cells $[21,22]$. In this scenario of malfunction of G1$\mathrm{S}$ checkpoint p53, c-Myc was a main factor accounting for ApoG2-induced S phase arrest. P21 and cyclins were followed by downregulation of c-Myc expression.

c-Myc is not only a central regulator of cell proliferation but also induces cells to undergo apoptosis, unless spe- cific signals provided by oncogenes block the apoptosis pathway [23]. Notably, NPC cells consistently harbor EBV DNA and express EBV proteins, LMP1 and BARF1; these proteins stimulate oncogenic antiapoptotic Bcl-2 proteins to protect host cancer cells from apoptosis [24-27]. ApoG2 is a potent inhibitor of antiapoptotic Bcl-2 proteins and its treatment could remove the protective effect of Bcl-2 proteins and facilitate apoptosis. In this case, downregulation of c-Myc expression by ApoG2 on one hand could let cells away from c-Myc-induced apoptosis and on other hand led to cell cycle arrest. However, by inhibiting Bcl-2 proteins, ApoG2 still helped release proapoptotic proteins, such as Bax and Bak, and irreversibly damaged mitochondria and induced cell apoptotic [5]. 
A.

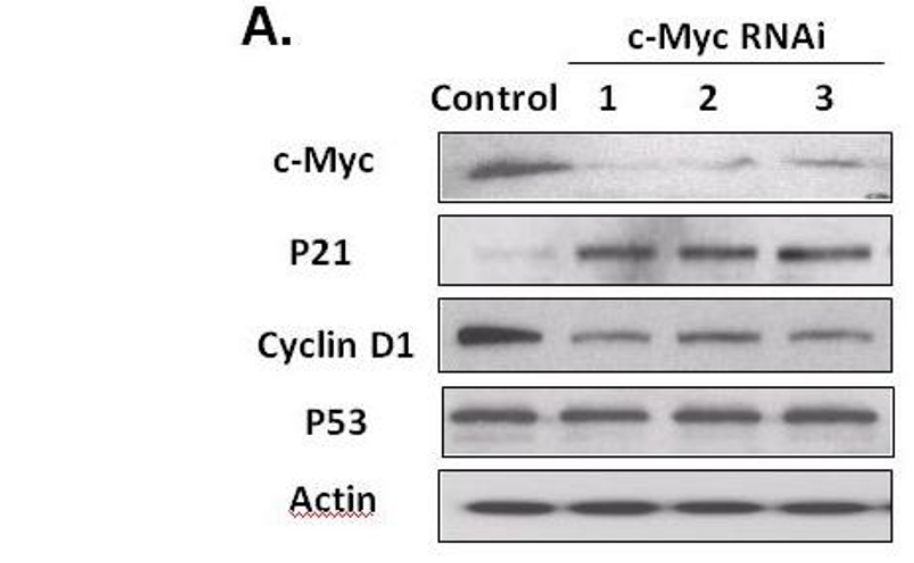

B.

C.
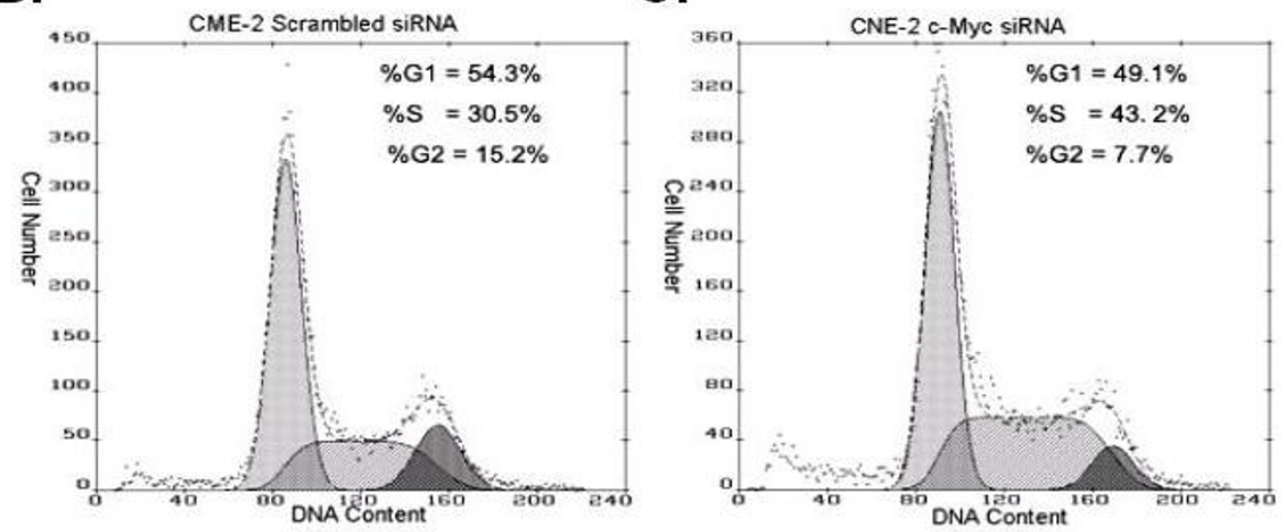

D.

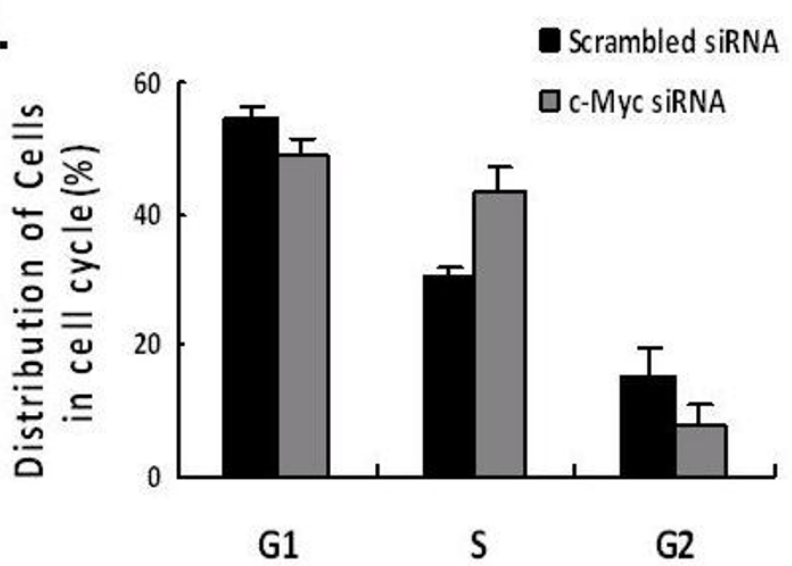

Figure 4

The effect c-Myc siRNA transfection on c-Myc downstream molecules and cell cycle distribution. (A) Comparison of the effect of c-Myc siRNA and scrambled (nontargeting) siRNA on the expression of c-Myc downstream molecules. Transfection of CNE-2 cells with c-Myc or scrambled (nontargeting) siRNA for $48 \mathrm{~h}$. Cells were then subjected to Western blotting using anti-c-Myc, -cyclin DI, -p2 I, and -p53 antibodies as described in Materials and Methods. Comparison of the effect of scrambled (nontargeting) siRNA (B) and c-Myc siRNA (C) on cell cycle distribution of CNE-2 cells using PI staining and flow cytometry. Each histogram is representative of three experiments. (D) Analysis of cell cycle distributions showed that, compared to srambled siRNA, c-Myc siRNA induced a conspicuous increasing of cells in S phase in CNE-2 cells at 48 h. Bar heights, average of three independent experiments; bars, SD. 
A.

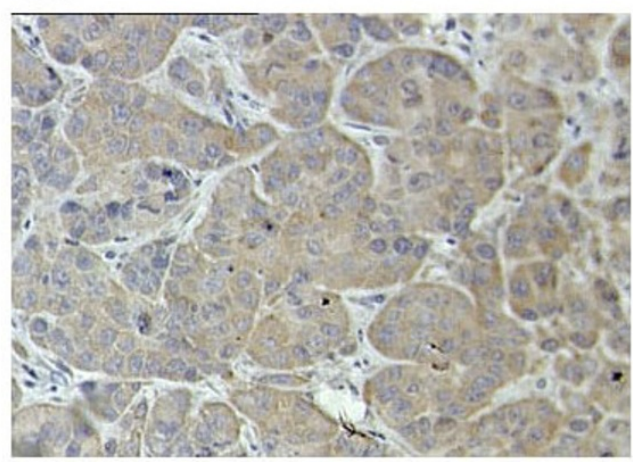

B.



C.

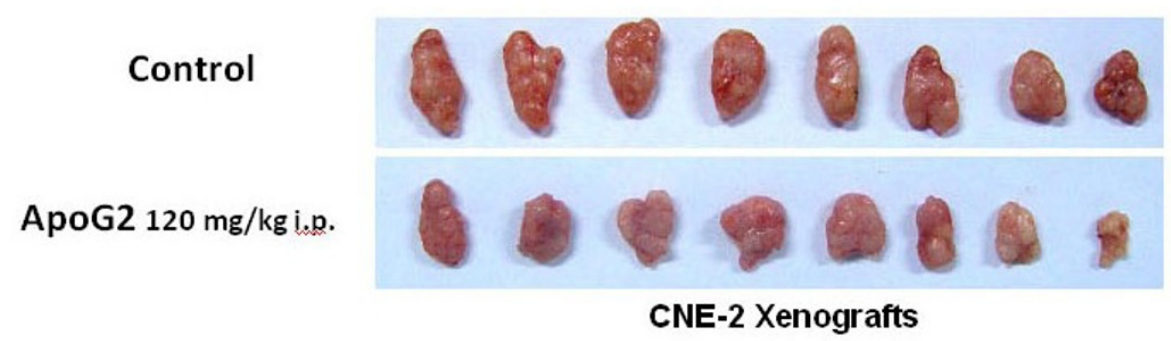

D.

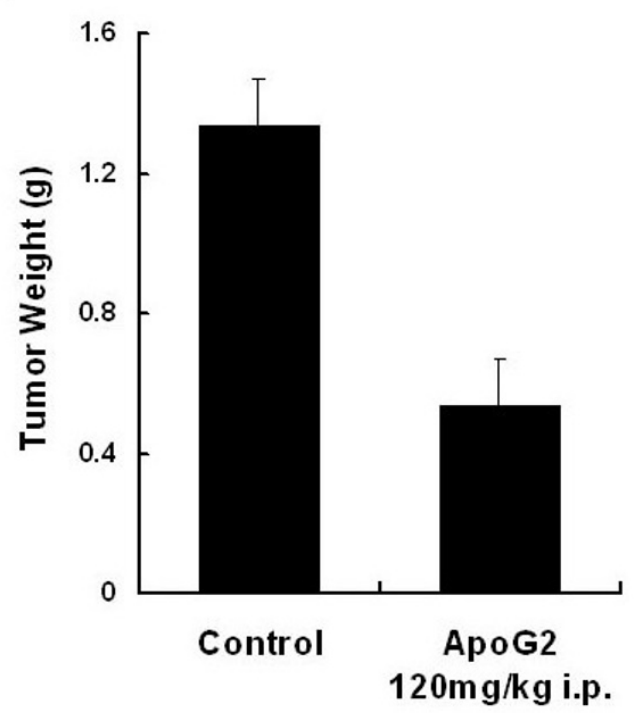

Figure 5

Analysis of the impact of ApoG2 in vivo on c-Myc expression and tumor growth in CNE-2 xenografts. The tumor tissues from ApoG2 ( $120 \mathrm{mg} / \mathrm{kg}$ intraperitoneal injection daily) treatment were obtained at the end of 12 days of treatment. Immunochemistry analysis of c-Myc expression in CNE-2 xenograft tumor sections after NS treatment (A) or ApoG2 treatment (B), magnification, $\times 80$. (C) Photographs of CNE-2 xenografts from NS treatment and ApoG2 treatment groups. When all tumors of the control group exceeded I $g$ in weight, the animal experiment was terminated and mice were killed. The tumors were taken out for weighing and comparing the effect of ApoG2 on tumors. (D) Antitumor acitivity of ApoG2 in CNE2 xenograft-bearing nude mice. Compared to control (NS treatment) mice, ApoG2 treatment greatly suppressed tumor weight. Bar heights, average weight of eight CNE-2 xenograft tumors; bars, SD. 
Gossypol is clinically used in China to treat adenomyosis and hysteromyoma because of its ability to inhibit estrogen and progesterone by competitively binding to the estrogen receptor and progesterone receptor [28]. c-Myc is a well-established target of estrogen action and plays a role in controlling cell cycle progression. Anti-estrogen treatment is reported to be able to cause an acute decrease in c-Myc expression, a subsequent decline in cyclin D1 expression, and, ultimately, inhibition of DNA synthesis and arrest of cells in a quiescent state [29]. Estrogen receptor and progesterone receptor are known to be highly expressed in NPC cells, and their expression is considered a sign of distant metastasis and a poor prognosis [30]. Based on our findings, we suggest that ApoG2-induced cell cycle arrest is dependent on ApoG2's downregulation of c-Myc expression. Use of ApoG2 to treat NPC may suppress the activity of estrogen and progesterone and reduce the incidence of distant metastasis and local relapse.

The concept of targeted biological therapy for cancer has emerged over the past decade. Clinical trials studying the efficacy and tolerability of these targeted agents has shown that most tumors depend on more than one signaling pathway for their growth and survival. Therefore, investigators pursue different strategies to inhibit multiple signaling pathways by developing multitargeted agents [31]. The recent U.S. Food and Drug Administration approval of sorafenib and sunitinib, which target vascular endothelial growth factor receptor, platelet-derived growth factor receptor, FLT-3, and c-Kit, marks the use of a new generation of multitarget anticancer drugs [32]. Our study show that ApoG2 is one such multitarget agent that targets both the antiapoptotic and cell cycle progression pathway in NPC cells by blocking antiapoptotic Bcl-2 proteins and the c-Myc oncogenic pathway. These findings provide an entirely new concept for the use of ApoG2 in cancer therapy.

\section{Conclusion}

Our findings indicated that ApoG2 can potently disturb the proliferation of NPC cells by suppressed c-Myc signaling pathway. This data suggested that the inhibitory effect of ApoG2 on NPC cell cycle proliferation might contribute to its use in anticancer therapy.

\section{Abbreviations}

ApoG2: apogossypolone; DMSO: dimethyl sulfoxide; EBV: Epstein-Barr virus; LMP1: latent membrane protein 1; MTT: (3-[4,5-dimethylthiazol-2-thiazolyl]-2,5-diphenyltetrazolium bromide; NPC: nasopharyngeal carcinoma; OD: optical density; PBS: phosphate-buffered saline; Rb: retinoblastoma gene; siRNA: small interfering RNA; TGF- $\beta 1$ : transforming growth factor- $\beta 1$.

\section{Competing interests}

The authors declare that they have no competing interests.

\section{Authors' contributions}

YXZ was responsible for study design. DY and XFZ performed the experiments and drafted the manuscript. JS participated in the data analysis and western-blot. All authors read and approved the final manuscript.

\section{Acknowledgements}

We thank Mr. Xiongwen Zhang (Director, Pharmacology, Ascenta Shanghai R \& D Center) for help with the drug preparation and Mr. Qing-Yu Kong (Department of Nephrology of the First Affiliated Hospital of Sun Yat-Sen University) for help with the flow cytometry.

\section{References}

I. McDermott AL, Dutt SN, Watkinson JC: The aetiology of nasopharyngeal carcinoma. Clin Otolaryngol Allied Sci 200I, 26:82-92.

2. Chan AT, Teo PM, Huang DP: Pathogenesis and treatment of nasopharyngeal carcinoma. Semin Oncol 2004, 3 I:794-80I.

3. Kwong DL, Sham JS, Au GK, Chua DT, Kwong PW, Cheng AC, Wu PM, Law MW, Kwok CC, Yau CC, et al.: Concurrent and adjuvant chemotherapy for nasopharyngeal carcinoma: a factorial study. I Clin Oncol 2004, 22:2643-2653.

4. Wang TL, Tan YO: Cisplatin and 5-fluorouracil continuous infusion for metastatic nasopharyngeal carcinoma. Ann Acad Med Singapore 1991, 20:601-603.

5. Hu ZY, Zhu XF, Zhong ZD, Sun J, Wang J, Yang D, Zeng YX: ApoG2, a novel inhibitor of antiapoptotic Bcl-2 family proteins, induces apoptosis and suppresses tumor growth in nasopharyngeal carcinoma xenografts. Int J Cancer 2008, I 23:24 I 8-2429.

6. Balakrishnan K, Wierda WG, Keating MJ, Gandhi V: Gossypol, a BH3 mimetic, induces apoptosis in chronic lymphocytic leukemia cells. Blood 2008, I I 2: I 97 |-1980.

7. Teng CS, Vilagrasa X: Biphasic c-Myc protein expression during gossypol-induced apoptosis in rat spermatocytes. Contraception 1998, 57:117-123.

8. Huang YW, Wang LS, Chang HL, Ye W, Sugimoto Y, Dowd MK, Wan PJ, Lin YC: Effects of serum on (-)-gossypol-suppressed growth in human prostate cancer cells. Anticancer Res 2006, 26:3613-3620.

9. Zhang M, Liu H, Guo R, Ling Y, Wu X, Li B, Roller PP, Wang S, Yang $D$ : Molecular mechanism of gossypol-induced cell growth inhibition and cell death of HT-29 human colon carcinoma cells. Biochem Pharmacol 2003, 66:93-103.

10. Chang JS, Hsu YL, Kuo PL, Chiang LC, Lin CC: Upregulation of Fas/ Fas ligand-mediated apoptosis by gossypol in an immortalized human alveolar lung cancer cell line. Clin Exp Pharmacol Physiol 2004, 3 I:71 6-722.

II. Zheng H, Li LL, Hu DS, Deng XY, Cao Y: Role of Epstein-Barr virus encoded latent membrane protein $I$ in the carcinogenesis of nasopharyngeal carcinoma. Cell Mol Immunol 2007, 4:185-196.

12. Voortman J, Checinska A, Giaccone G, Rodriguez JA, Kruyt FA: Bortezomib, but not cisplatin, induces mitochondria-dependent apoptosis accompanied by up-regulation of noxa in the nonsmall cell lung cancer cell line NCl-H460. Mol Cancer Ther 2007, 6:1046-1053

13. Guo C, Pan ZG, Li DJ, Yun JP, Zheng MZ, Hu ZY, Cheng LZ, Zeng $Y X$ : The expression of $p 63$ is associated with the differential stage in nasopharyngeal carcinoma and EBV infection. J Transl Med 2006, 4:23.

14. Gilbert NE, O'Reilly JE, Chang CJ, Lin YC, Brueggemeier RW: Antiproliferative activity of gossypol and gossypolone on human breast cancer cells. Life Sci 1995, 57:6I-67.

I5. Gartel AL, Shchors K: Mechanisms of c-myc-mediated transcriptional repression of growth arrest genes. Exp Cell Res 2003, 283:17-2I. 
16. Fan CS, Wong N, Leung SF, To KF, Lo KW, Lee SW, Mok TS, Johnson PJ, Huang DP: Frequent c-myc and Int-2 overrepresentations in nasopharyngeal carcinoma. Hum Pathol 2000, 31:169-178.

17. Lemaire P, Livingstone DR: Aromatic hydrocarbon quinonemediated reactive oxygen species production on hepatic microsomes of the flounder (Platichthys flesus L.). Comp Biochem Physiol C Pharmacol Toxicol Endocrinol 1997, I 17:131-139.

18. Trachootham D, Zhou Y, Zhang H, Demizu Y, Chen Z, Pelicano H, Chiao PJ, Achanta G, Arlinghaus RB, Liu J, Huang P: Selective killing of oncogenically transformed cells through a ROS-mediated mechanism by beta-phenylethyl isothiocyanate. Cancer Cell 2006, 10:24I-252

19. Antosiewicz J, Ziolkowski W, Kar S, Powolny AA, Singh SV: Role of reactive oxygen intermediates in cellular responses to dietary cancer chemopreventive agents. Planta Med 2008, 74:1570-1579.

20. Van Poznak C, Seidman AD, Reidenberg MM, Moasser MM, Sklarin N, Van Zee K, Borgen P, Gollub M, Bacotti D, Yao TJ, et al.: Oral gossypol in the treatment of patients with refractory metastatic breast cancer: a phase I/II clinical trial. Breast Cancer Res Treat 200I, 66:239-248.

21. Szekely L, Selivanova G, Magnusson KP, Klein G, Wiman KG: EBNA5 , an Epstein-Barr virus-encoded nuclear antigen, binds to the retinoblastoma and p53 proteins. Proc Natl Acad Sci USA 1993, 90:5455-5459.

22. Crook T, Nicholls JM, Brooks L, O'Nions J, Allday MJ: High level expression of deltaN-p63: a mechanism for the inactivation of p53 in undifferentiated nasopharyngeal carcinoma (NPC)? Oncogene 2000, 19:3439-3444.

23. Desbarats L, Schneider A, Muller D, Burgin A, Eilers M: Myc: a single gene controls both proliferation and apoptosis in mammalian cells. Experientia 1996, 52:1।23-II29.

24. Henderson S, Rowe M, Gregory C, Croom-Carter D, Wang F, Longnecker R, Kieff $E$, Rickinson A: Induction of bcl-2 expression by Epstein-Barr virus latent membrane protein I protects infected B cells from programmed cell death. Cell 1991, 65: $1107-1115$.

25. Sheng W, Decaussin G, Sumner S, Ooka T: $\mathbf{N}$-terminal domain of BARFI gene encoded by Epstein-Barr virus is essential for malignant transformation of rodent fibroblasts and activation of BCL-2. Oncogene 200I, 20: I I76-I I85.

26. $D$ 'Souza $B$, Rowe $M$, Walls $D$ : The bfl-I gene is transcriptionally upregulated by the Epstein-Barr virus LMPI, and its expression promotes the survival of a Burkitt's lymphoma cell line. J Virol 2000, 74:6652-6658.

27. Kawanishi M: Expression of Epstein-Barr virus latent membrane protein I protects Jurkat $T$ cells from apoptosis induced by serum deprivation. Virology 1997, 228:244-250.

28. Zhang YW, Han ML, Wang YF: [Estrogen and progesterone cytosol receptor concentrations in patients with endometriosis and their changes after gossypol therapy]. Zhonghua $\mathrm{Fu}$ Chan Ke Za Zhi 1994, 29:220-223. 253

29. Wosikowski K, Kung W, Hasmann M, Loser R, Eppenberger U: Inhibition of growth-factor-activated proliferation by anti-estrogens and effects on early gene expression of MCF-7 cells. Int j Cancer 1993, 53:290-297.

30. Xu B, Hu P, Wu Q, Hou ], Zhang B, Chen X, Huang G: [Relationship between expression of estrogen receptor progestrone receptor and the biological characteristics of nasopharyngeal carcinoma]. Lin Chuang Er Bi Yan Hou Ke Za Zhi 1999, 13:347-349.

31. Petrelli A, Giordano S: From single- to multi-target drugs in cancer therapy: when aspecificity becomes an advantage. Curr Med Chem 2008, I 5:422-432.

32. Sartore-Bianchi A, Ricotta R, Cerea G, Maugeri MR, Siena S: Rationale and clinical results of multi-target treatments in oncology. Int J Biol Markers 2007, 22:S77-87.

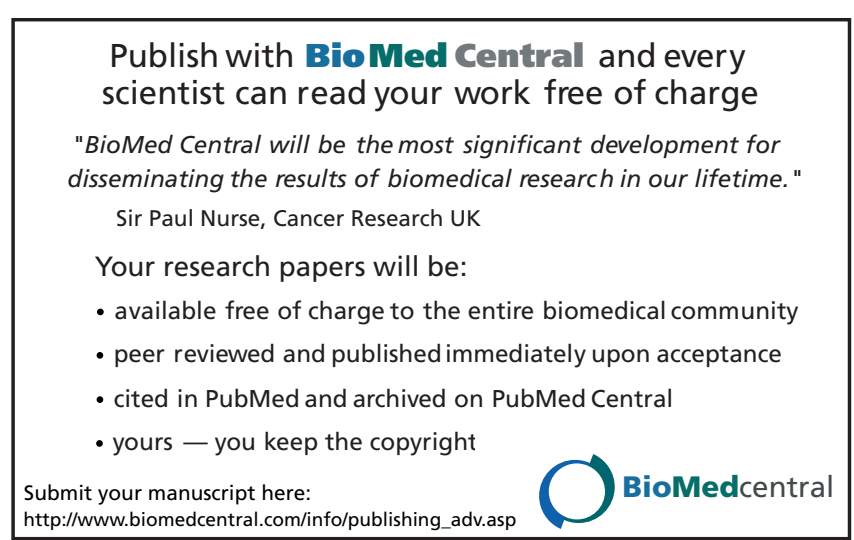

